\title{
Neurocysticercosis: An Easy to Miss Diagnosis in Non-Endemic Regions
}

\author{
Akshit Chitkara ${ }^{1}$, Sarah Chhabra ${ }^{2}$, Akshit Griwan ${ }^{3}$, Naman Khurana ${ }^{4}$, Piyush Puri ${ }^{5}$ \\ 1. Internal Medicine, Sh. Moolchand Super Speciality Hospital, Karnal, IND 2. Internal Medicine, Mount Sinai Medical \\ Center, Miami, USA 3. Internal Medicine, East Tennessee State University Quillen College of Medicine, Mountain \\ Home, USA 4. Obstetrics and Gynecology, Sanjay Gandhi Hospital, Delhi, IND 5. Internal Medicine, Al Flah School of \\ Medical Sciences and Research Center, Faridabad, IND
}

Corresponding author: Akshit Chitkara, akshit.chitkara6@gmail.com

\begin{abstract}
A 29-year-old male presented with swollen gums and stomatitis for the past two months. History revealed that he had moved to the United States from India six years ago and had a first episode of generalized tonicclonic seizure with confusion and loss of consciousness. Meningioma of the brain was diagnosed, and a Gamma Knife excision of the meningioma was planned. The patient refused to proceed with the surgery and came back to India for a second opinion. Upon repeat MRI scan, the neurosurgeon revised the diagnosis to neurocysticercosis (NCC), and the patient was treated with albendazole, prednisolone, and phenytoin and recovered completely. Hence an unnecessary brain surgery was avoided. The complaint of stomatitis and gingival hypertrophy was due to the side effects of phenytoin.
\end{abstract}

NCC remains a major public health problem in developing countries, and it should be considered as a differential diagnosis in patients from NCC endemic regions.

Categories: Internal Medicine, Neurology, Neurosurgery

Keywords: neuro-surgery, neuro-imaging

\section{Introduction}

Neurocysticercosis (NCC) is the most severe form of cysticercosis, an infection caused by ingesting tapeworm (Taenia solium) eggs. The larvae may travel to the brain via the bloodstream and develop into NCC. It is a very common disorder in the Indian subcontinent, Latin America, Africa, and China (1:1000) and is responsible for about $30 \%$ of total cases of epilepsy [1-3]. In developed countries, it is a comparatively rare disorder, so it is more often misdiagnosed or missed while making the differential diagnosis [4].

Review began $11 / 28 / 2020$ Review ended 12/06/2020 Published 12/13/2020

\section{(c) Copyright 2020}

Chitkara et al. This is an open access article distributed under the terms of the Creative Commons Attribution License CC-BY 4.0., which permits unrestricted use, distribution, and reproduction in any medium, provided the original author and source are credited.
The radiological (MRI/contrast-enhanced CT) features of NCC are quite similar to those of brain abscess, tuberculoma, meningioma, brain metastasis, or hydatid cyst, leading to wrong interpretation of the brain lesion [5]. Hence the geographic background and travel history of the patient must be evaluated while analyzing the lab work-up and neuroimaging data.

\section{Case Presentation}

A 29-year-old male presented with swollen gums and stomatitis for the past two months. The patient denied throat pain, fever, upper respiratory tract infection (URTI), diarrhea, dysentery, abdominal pain, or any urinary problem. There was no history of diabetes mellitus, hypertension, or coronary artery disease. He was a non-smoker and non-alcoholic. There was no history of recreational drug use or tobacco chewing. He consumed mildly spicy food and around two cups of tea or coffee per day. He was obese with a BMI of 28 .

On further interrogation, the patient revealed that six years ago, when he was 23 years old, he had moved to the US, from India, for higher education. Eight months after his move, he suffered a generalized tonic-clonic seizure with loss of consciousness that lasted for 10 minutes. Upon a 911 call, he was immediately hospitalized, and fosphenytoin and prednisolone were started. After regaining consciousness, the patient had confusion but no neurological deficit. After laboratory workup and neuroimaging, a final diagnosis of meningioma of the right frontal lobe of the brain was established, and surgical resection was planned (Figures 1-3). 


\section{Cureus}

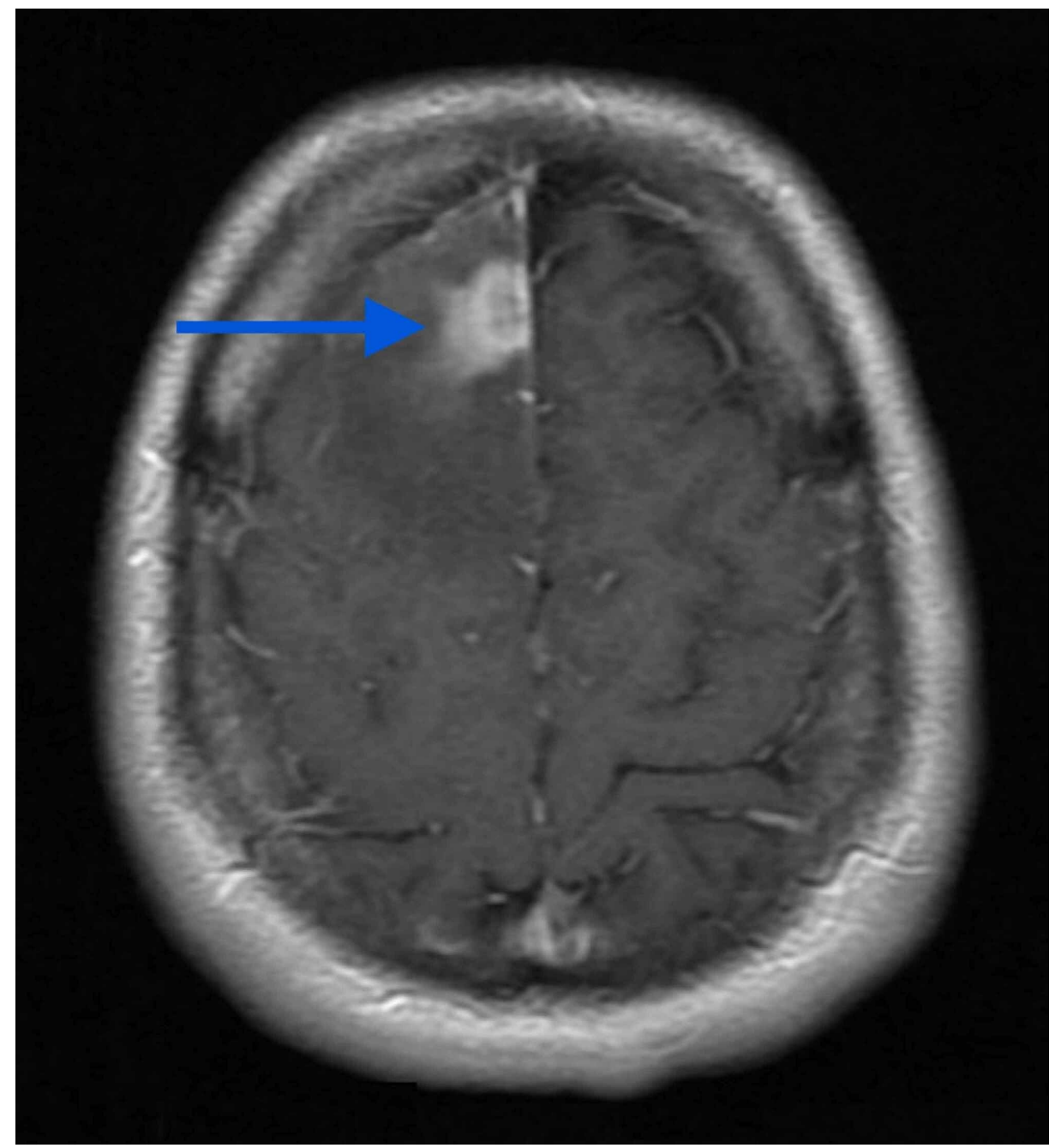

FIGURE 1: Axial post-contrast T1 MRI showing $16 \times 14 \times 21 \mathrm{~mm}$ conglomerate ring-enhancing lesion in the paramedian region of the right frontal lobe with surrounding edema. 


\section{Cureus}

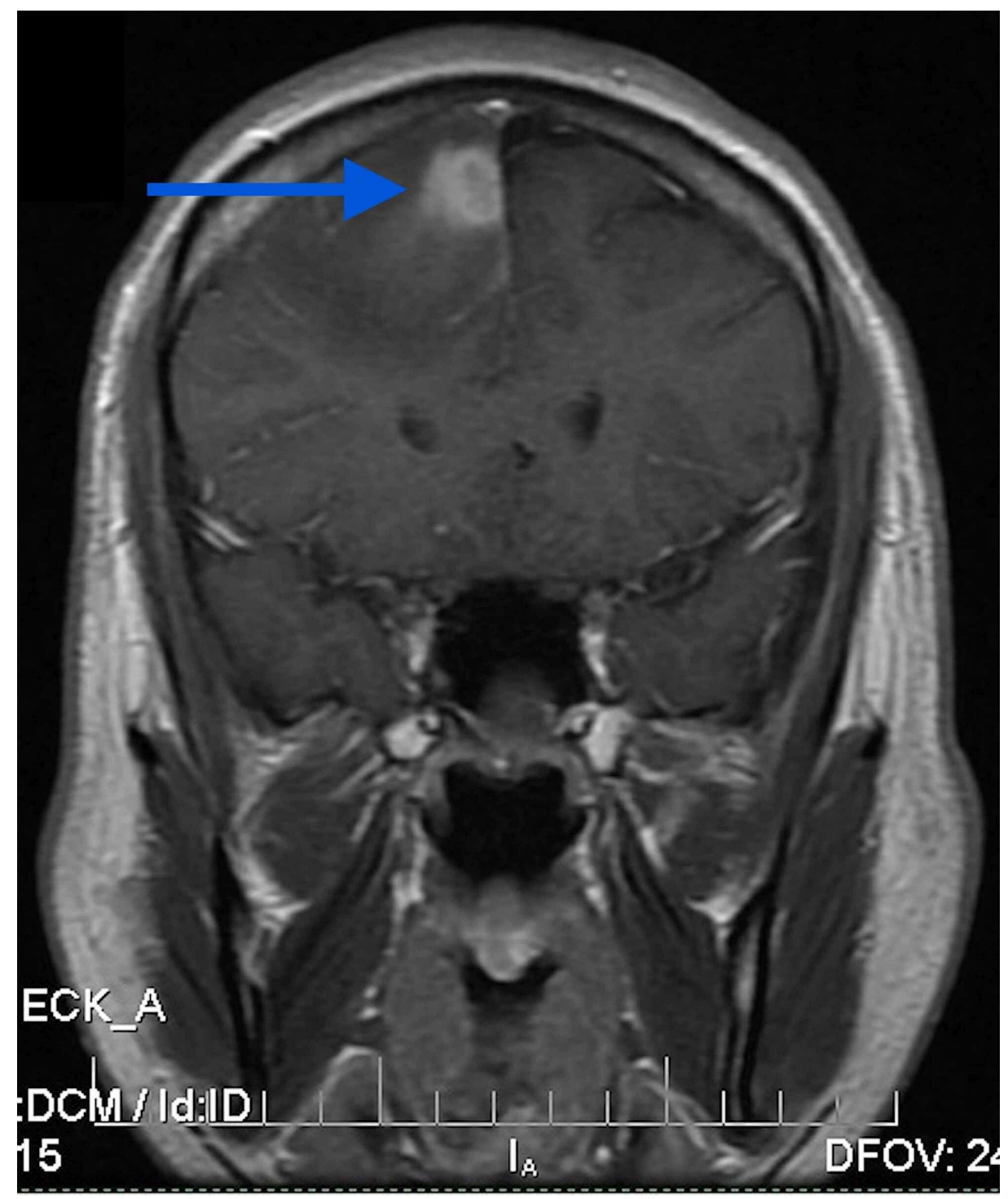

FIGURE 2: Coronal post-contrast T1 MRI showing 16×14×21 mm conglomerate ring-enhancing lesion in the paramedian region of the right frontal lobe with surrounding edema. 


\section{Cureus}

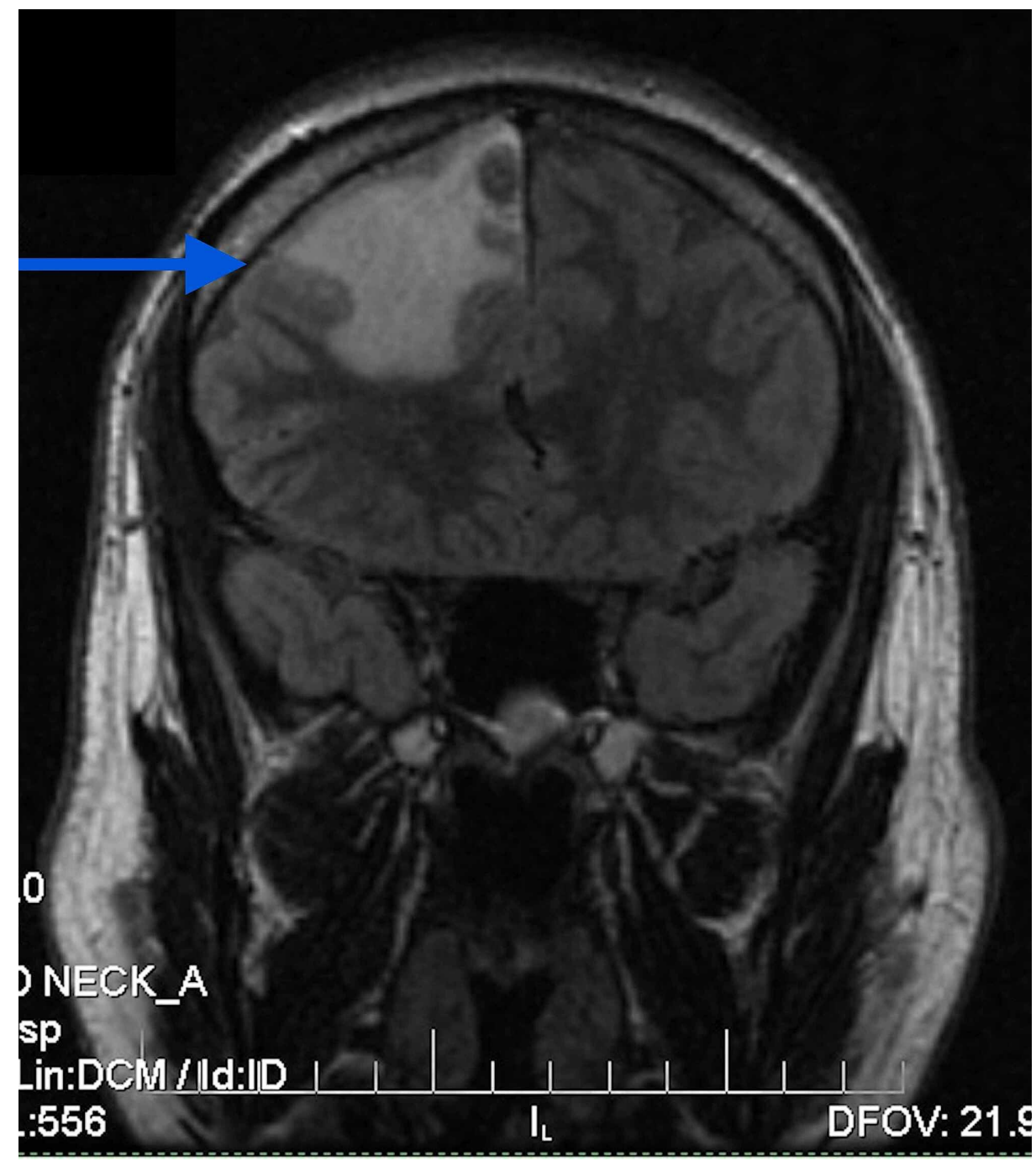

FIGURE 3: Coronal T2 FLAIR MRI showing $16 \times 14 \times 21 \mathrm{~mm}$ conglomerate ring-enhancing lesion in the paramedian region of the right frontal lobe with surrounding edema.

FLAIR: fluid-attenuated inversion recovery

The patient refused to proceed with the surgery and came back to India after two weeks for a second opinion. The neurosurgeon in India reordered neuroimaging. To confirm the diagnosis, two MRIs were conducted by two separate radiologists. They compared the older scan with the new MRI studies and revised the diagnosis to NCC (Figures 4-6). 


\section{Cureus}

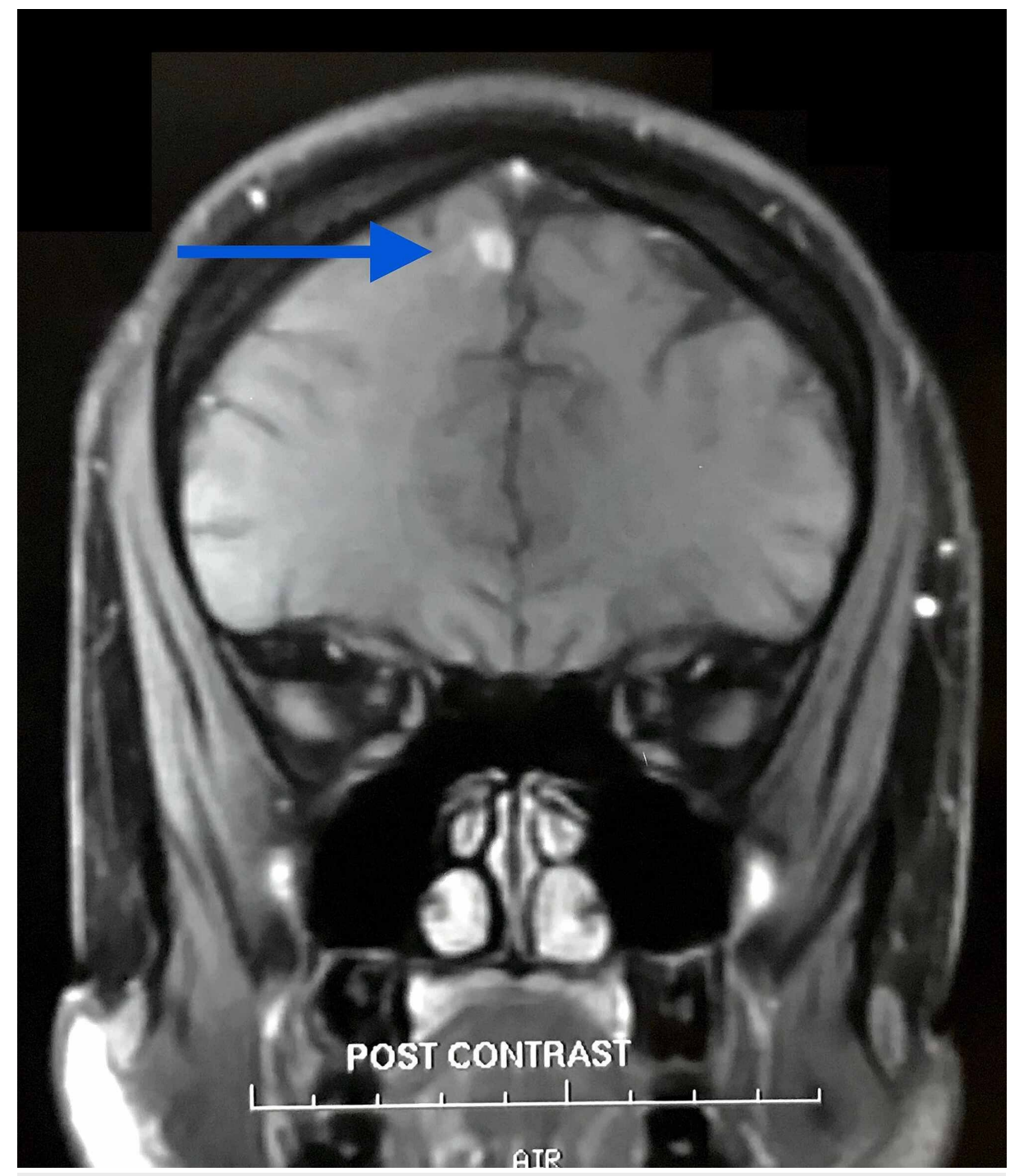

FIGURE 4: Neurocysticercosis in the right frontal lobe of the brain, postcontrast coronal MRI. 


\section{Cureus}

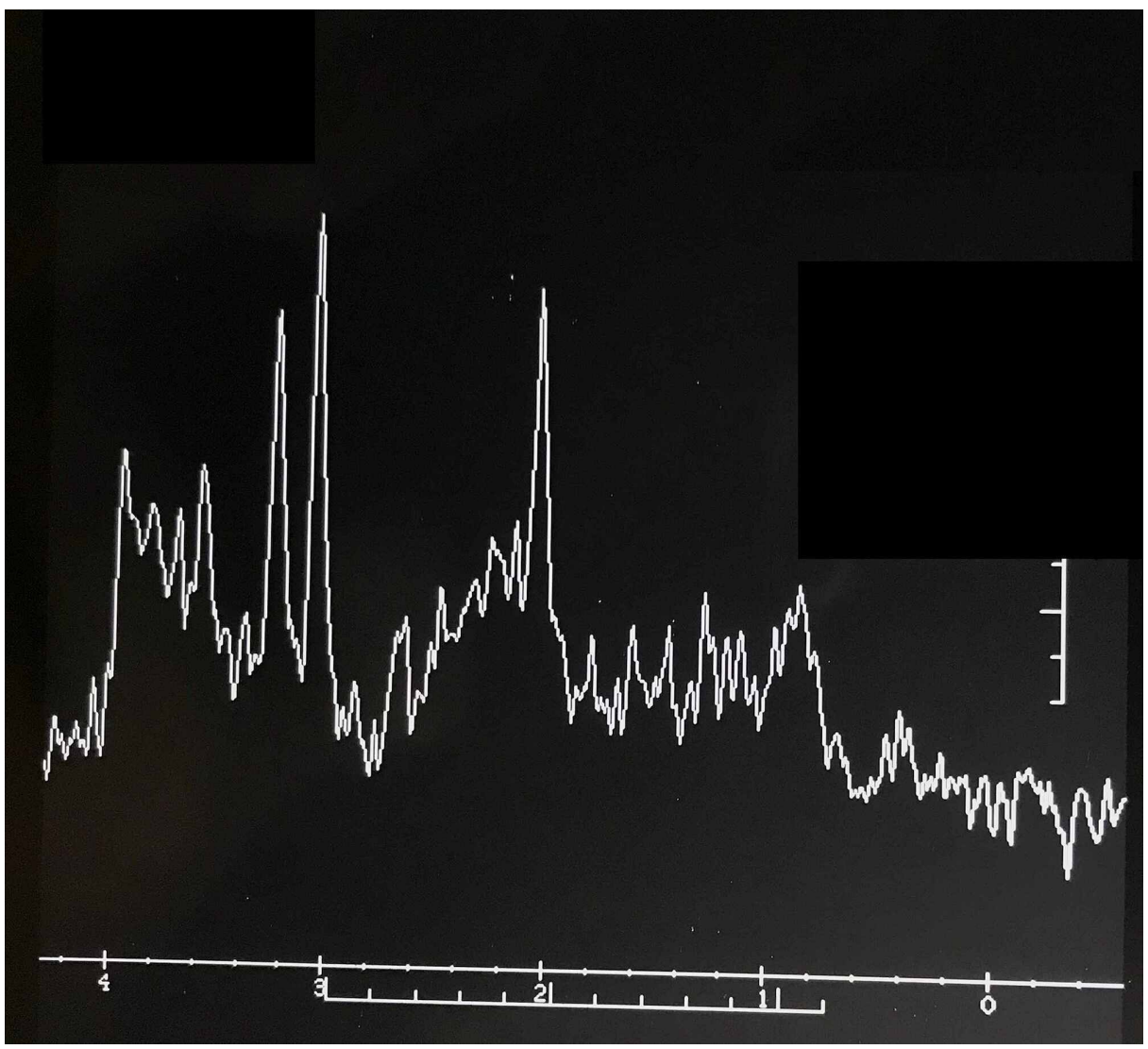

FIGURE 5: MR spectroscopy through the lesion shows normal Creatine (Cr), Choline (cho), and $\mathrm{N}$-acetylaspartate (Naa) ratio with no abnormal increase in Myo-inositol (MI) or any lipid or lactate peak. 


\section{Cureus}

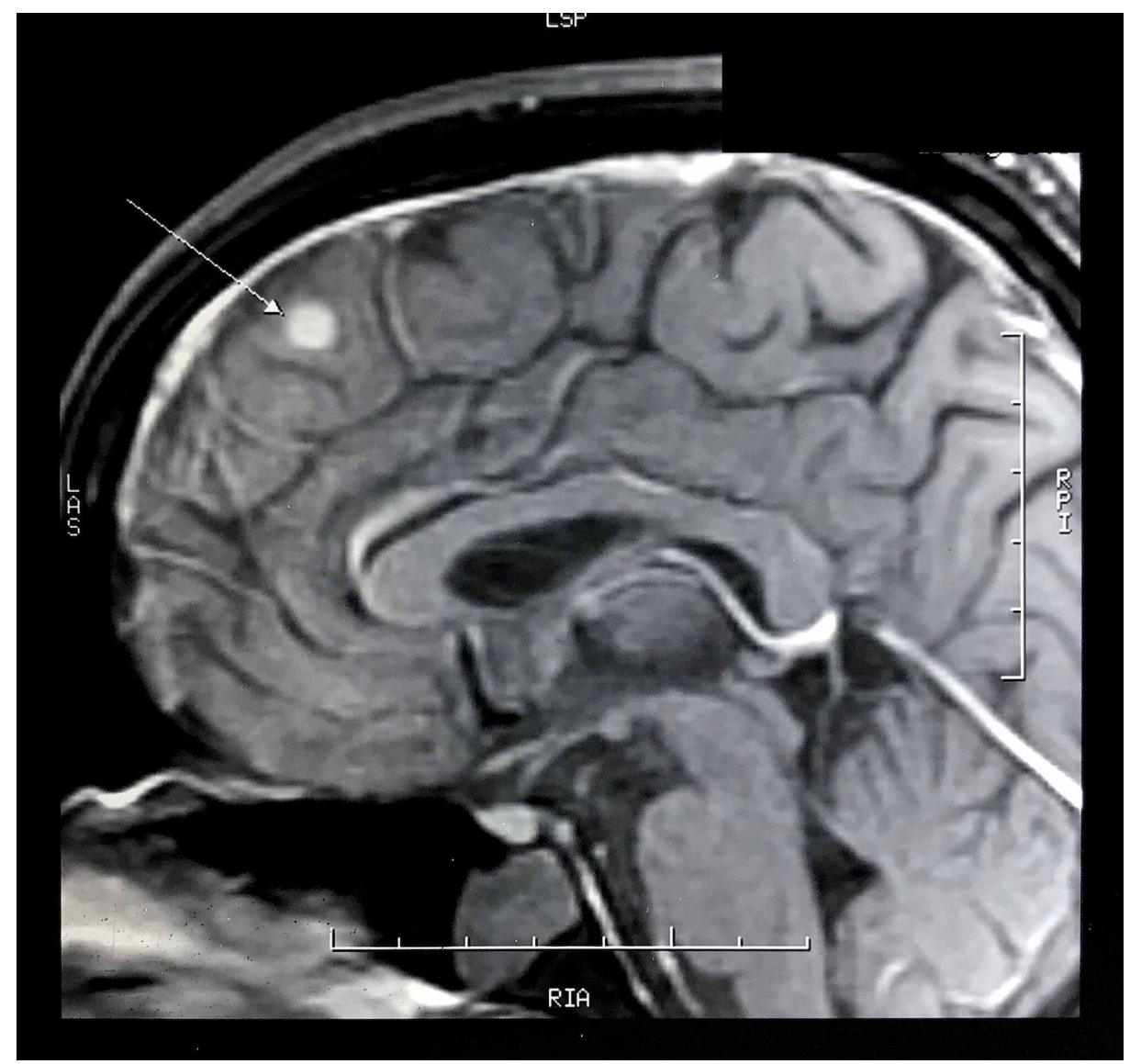

FIGURE 6: Neurocysticercosis in the right frontal lobe of the brain on MRI.

\section{Investigations}

MRI study of the US hospital showed a $16 \times 14 \times 21 \mathrm{~mm}$ conglomerate ring-enhancing lesion in the paramedian region of the right frontal lobe with surrounding edema (Figures 1-3).

MRI study done in India revealed nodular intraparenchymal disc enhancing lesions in the paramedian region of the right frontal lobe, $7 \times 7 \times 6 \mathrm{~mm}$ in size. It was hypointense on $\mathrm{T} 2$ weighted image (T2WI), and a moderate degree of edema was noted around the lesion. There was no midline shift. The final impression confirmed the presence of a degenerating NCC in the right frontal lobe of the brain (Figures 4, 6).

Laboratory results for the complaints of gingivitis were as follows: Haemoglobin $14.4 \mathrm{~g} / \mathrm{dL}$, white blood cells 5,700 per microliter, platelet count 242,000 per microliter, neutrophil $57 \%$, basophil $0.4 \%$, lymphocyte $36.6 \%$, monocyte $5.3 \%$, eosinophil 0.7\%, blood glucose $122 \mathrm{mg} / \mathrm{dL}$, blood urea nitrogen (BUN) $10 \mathrm{mg} / \mathrm{dL}$, serum creatinine $1.02 \mathrm{mg} / \mathrm{dL}$, serum calcium $9.6 \mathrm{mg} / \mathrm{dL}$, alkaline phosphatase $64 \mathrm{U} / \mathrm{L}$, serum phenytoin level $<1.0 \mathrm{mcg} / \mathrm{ml}$

\section{Differential diagnosis}

Establishing the diagnosis of NCC is not always a straightforward process. NCC, brain abscess, tuberculoma, hydatid cyst, malignancy, fungal infections, syphilis, sarcoidosis, or brain metastasis may look alike on neuroimaging in the form of small nodular intraparenchymal disc enhancing lesion [5]. Hence the diagnosis may be difficult even with neuroimaging and serological studies.

NCC is the most common parasitic disease of the central nervous system in the Indian subcontinent along with Latin America, Africa, and China [2,3]. Therefore geographic background and travel history of the patient must be taken into account before finalizing the diagnosis for such patients.

\section{Outcome and follow-up}

There are multiple side effects of phenytoin therapy $[6,7]$. The patient has been asymptomatic and seizurefree since the initial occurrence. 


\section{Discussion}

Approximately $25 \%$ of cases of epilepsy in India are due to NCC [8]. In 2011, it was estimated that NCC associated active epilepsy led to an annual median loss of US $\$ 185.14$ million, leading to significant health and economic impact in India [9]. The pork tapeworm (Taenia solium) infects the human nervous system and skeletal muscles. These larvae enter the bloodstream and settle down in the brain, retina, subcutaneous tissue, or skeletal muscle. The larvae evoke a cellular reaction starting with infiltration of neutrophils, eosinophils, lymphocytes, plasma cells, and at times giant cells. It produces a granuloma and may remain alive for multiple years [10]. They may die, and the lesion may get calcified. In addition to epilepsy, the granuloma may lead to a focal neurological deficit, visual disturbances, behavioral disorders, or obstructive hydrocephalus.

This disease is one of the main causes of epileptic seizures in many less developed countries. It is also increasingly seen in more developed countries because of immigration from endemic areas. NCC is extremely prevalent in Latin America, the Indian subcontinent, Sub-Saharan Africa, coastal North Africa, and China [2,3]. Open field defecation, dirty toilets, poor sanitation are the main culprits for the excessive prevalence of this disease [11].

The lesions in the brain on neuroimaging may resemble those of hydatid cyst, tuberculoma, meningioma, brain abscess, or brain metastasis. Eosinophilia in blood or cerebrospinal fluid (CSF) and enzyme-linked immunosorbent assay (ELISA) testing may help, but neuroimaging is the mainstay for diagnosing NCC [12].

NCC usually does not require any surgery. Albendazole $15 \mathrm{mg}$ per $\mathrm{kg}$ per day or praziquantel $50 \mathrm{mg}$ per $\mathrm{kg}$ per day for two to four weeks are very good larvicidal drugs [13]. Prednisolone $30 \mathrm{mg}$ per day for two to four weeks helps in reducing the inflammatory edema around the lesion and may be tapered gradually over the next two to three weeks [14]. Antihelminthic treatment, together with anticonvulsants, can reduce seizure activity by greater than $90 \%$, and anticonvulsants should be given for a minimum of three years, extendable to five years or lifetime, subject to recovery and seizure-free period [15].

\section{Conclusions}

Detailed history taking is the most important part of any patient's workup. Personal history, family history, history of travel, and immigration are very important in today's culturally diverse and fast-moving world, and more so when infective pathology is a part of the differential diagnosis. By being more vigilant and thorough, we can safeguard the patients from unnecessary surgical procedures and their sequelae. Side effects such as gum hypertrophy should not deter a physician from using phenytoin in the treatment of epilepsy. There should be proper monitoring of the phenytoin levels to avoid its toxicity as it has a narrow therapeutic index and wide dose-related side effect profile.

\section{Additional Information \\ Disclosures}

Human subjects: Consent was obtained by all participants in this study. Conflicts of interest: In compliance with the ICMJE uniform disclosure form, all authors declare the following: Payment/services info: All authors have declared that no financial support was received from any organization for the submitted work. Financial relationships: All authors have declared that they have no financial relationships at present or within the previous three years with any organizations that might have an interest in the submitted work. Other relationships: All authors have declared that there are no other relationships or activities that could appear to have influenced the submitted work.

\section{References}

1. Garcia HH, Nash TE, Del Brutto OH: Clinical symptoms, diagnosis, and treatment of neurocysticercosis. Lancet Neurol. 2014, 13:1202-15. 10.1016/S1474-4422(14)70094-8

2. Dumas M, Grunitzky E, Deniau M, et al.: Epidemiological study of neuro-cysticercosis in northern Togo (West Africa). Acta Leiden. 1989, 57:191-6.

3. Garcia-Noval J, Allan JC, Fletes C, et al.: Epidemiology of Taenia solium taeniasis and cysticercosis in two rural Guatemalan communities. Am J Trop Med Hyg. 1996, 55:282-9. 10.4269/ajtmh.1996.55.282

4. Kelesidis T, Tsiodras S: Extraparenchymal neurocysticercosis in the United States. Am J Med Sci. 2012, 344:79-82. 10.1097/MAJ.0b013e31823e6565

5. Omuro AM, Leite CC, Mokhtari K, Delattre JY: Pitfalls in the diagnosis of brain tumours . Lancet Neurol. 2006, 5:937-48. 10.1016/S1474-4422(06)70597-X

6. Dongari A, McDonnell HT, Langlais RP: Drug-induced gingival overgrowth. Oral Surg Oral Med Oral Pathol. 1993, 76:543-8. 10.1016/0030-4220(93)90027-2

7. Thomason JM, Seymour RA, Rawlins MD: Incidence and severity of phenytoin-induced gingival overgrowth in epileptic patients in general medical practice. Community Dent Oral Epidemiol. 1992, 20:288-91. 10.1111/j.1600-0528.1992.tb01701.x

8. Goel D, Dhanai JS, Agarwal A, Mehlotra V, Saxena V: Neurocysticercosis and its impact on crude prevalence rate of epilepsy in an Indian community. Neurol India. 2011, 59:37. 10.4103/0028-3886.76855

9. Singh BB, Khatkar MS, Gill JP, Dhand NK: Estimation of the health and economic burden of 


\section{Cureus}

neurocysticercosis in India. Acta Trop. 2017, 165:161-9. 10.1016/j.actatropica.2016.01.017

10. Dixon HB, Lipscomb FM: Cysticercosis: an analysis and follow-up of 450 cases . Proc R Soc Med. 1962, 55:242.

11. Prasad KN, Verma A, Srivastava S, Gupta RK, Pandey CM, Paliwal VK: An epidemiological study of asymptomatic neurocysticercosis in a pig farming community in northern India. Trans R Soc Trop Med Hyg. 2011, 105:531-6. 10.1016/j.trstmh.2011.06.001

12. Rosas N, Sotelo J, Nieto D: ELISA in the diagnosis of neurocysticercosis . Arch Neurol. 1986, 43:353-6. 10.1001/archneur.1986.00520040039016

13. Del Brutto OH, Roos KL, Coffey CS, García HH: Meta-analysis: cysticidal drugs for neurocysticercosis: albendazole and praziquantel. Ann Intern Med. 2006, 4:43-51. 10.7326/0003-4819-145-1-200607040-00009

14. Baird RA, Wiebe S, Zunt JR, Halperin JJ, Gronseth G, Roos KL: Evidence-based guideline: treatment of parenchymal neurocysticercosis: report of the Guideline Development Subcommittee of the American Academy of Neurology. Neurology. 2013, 80:1424-9. 10.1212/WNL.0b013e31828c2f3e

15. Vazquez V, Sotelo J: The course of seizures after treatment for cerebral cysticercosis . N Engl J Med. 1992, 327:696-701. 10.1056/NEJM199209033271005 